\title{
End-Effector Position Analysis Using Forward Kinematics for 5 DOF Pravak Robot Arm
}

\author{
Jolly Shah ${ }^{1}$, S.S.Rattan ${ }^{1}$, B.C.Nakra ${ }^{2}$ \\ ${ }^{1}$ ME Department, NIT, Kurukshetra, 136118, India \\ ${ }^{2}$ ME Department, ITM University, Gurgaon, 122001, India
}

\begin{tabular}{l} 
Article Info \\
\hline Article history: \\
Received Jan 1, 2013 \\
Revised May 4, 2013 \\
Accepted Jun 2, 2013 \\
\hline
\end{tabular}

Keyword:

Automatic control

D-H model

Forward kinematics

Robot manipulator

\begin{abstract}
Automatic control of the robotic manipulator involves study of kinematics and dynamics as a major issue. This paper involves the kinematic analysis of a Pravak Robot arm which is used for doing successful robotic manipulation task in its workspace. The Pravak Robot Arm is a 5-DOF robot having all the joints revolute. The kinematics problem is defined as the transformation from the Cartesian space to the joint space and vice versa. In this study the Denavit- Hartenberg (D-H) model is used to model robot links and joints. Pravak Robot Arm is a simple and safe robotic system designed for laboratory training and research applications. This robot allows to gain theoretical and practical experience in robotics, automation and control systems. The MATLAB R2007 is used to analyse end effectors position for a set of joint parameter.
\end{abstract}

Copyright $@ 2013$ Institute of Advanced Engineering and Science. All rights reserved.

\section{Corresponding Author:}

Jolly Shah,

Departement of Mechanical Engineering,

National Institute of Technology,

Kurukshetra, 136118, India.

Email: jollyshah80@yahoo.com

\section{INTRODUCTION}

Robot kinematics is the study of the motion of robotic mechanisms. Since the performance of specific manipulator tasks is achieved through the movement of the manipulator linkages, kinematics is of fundamental importance in robot design and control.

A kinematic equation provides the relationship between the joint displacement and the resulting endeffector position and orientation. The problem of finding the end-effector position and orientation for a given set of joint displacements is referred to as the forward kinematics problem. That is, the forward kinematics problem allows one to specify in a unique manner the relationship between the ( $\mathrm{n} \times 1)$ joint vector $\theta$ and the ( $\mathrm{m} \times 1)$ Cartesian vector $\mathrm{x}$ as:

$$
x(t)=f(\theta(t))
$$

Where $\mathrm{f}$ is the function defining the forward kinematic relation of the manipulator. Normally, the forward Kinematic equation can be obtained from the spatial geometry of the manipulator or by solving certain matrix algebraic equations. As the number of degrees of freedom (n) increases, the kinematic equation becomes more complex. Hence, the amount of computation required to compute the end-effectors position can become quite large.

The inverse kinematics problem consists of the determination of the joint variables corresponding to a given end-effectors orientation and position and is given by the equation, 
$\theta(t)=f-1(x(t))$

In a kinematic analysis, the position, velocity, and acceleration of all the links are calculated with respect to a fixed reference coordinate system, without considering the forces or moments.

The kinematic models are needed for off-line and on-line program generation and for tracking functional trajectories. A robotic manipulator is designed to perform a task in the 3-D space. The tool or endeffector is required to follow a planned trajectory to manipulate objects.

\section{KINEMATIC MODEL OF PRAVAK ROBOT ARM}

Pravak robot arm comprises of the 5-DOF with 3-DOF at the links and 2-DOF at the wrist.



Figure 1. Representation of Pravak Robot Arm

The diagrammatic representation of the Pravak robot arm is shown in Figure 1. The Pravak make robot has five joints. These joints perform the following motions as described in Table 1.

Table 1. Motions of various joints

\begin{tabular}{ccc}
\hline Joint No. & Motion & Movement \\
\hline Joint 0 & Waist & Left/Right \\
Joint 1 & Shoulder & Forward/Backward \\
Joint 2 & Elbow & Up/Down \\
Joint 3 & Wrist Pitch & Sky turn/Earth turn \\
Joint 4 & Wrist Roll & Clock/Anti-clock \\
\hline
\end{tabular}

There are six motors, five of which are used to move the joints while the sixth motor opens and closes the gripper, which is not treated as a joint. The center point of the gripper fingers is known as the tool tip or tool centroid and any movement that changes the position or the orientation of the centroid is a joint movement. Thus, it is obvious that the opening and the closing of the gripper is not a joint.

\subsection{Denavit \& Hartenberg (D-H) notation}

The definition of a manipulator with four joint-link parameters for each link and a systematic procedure for assigning right-handed orthonormal coordinate frames, one to each link in an open kinematic chain, was proposed by Denavit \& Hartenberg ,so is known as Denavit -Hartenberg (DH) notation. Figure 2 shows a pair of adjacent links, link(i-1) and link $\mathrm{i}$, their associated joints, joint (i-1), $\mathrm{i}$ and $(\mathrm{i}+1)$, and axis ( $\mathrm{i}-$ 2), (i-1) and i respectively.

A frame $\{i\}$ is assigned to link $i$ as follows:

i. The $\mathrm{Zi}-1$ lies along the axis of motion of the ith joint.

ii. The $\mathrm{Xi}$ axis is normal to the $\mathrm{Zi}-1$ axis, and pointing away from it.

iii. The Yi axis completes the right - handed coordinate system as required.

The DH representation of a rigid link depends on four geometric parameters associated with each links. These four parameters completely describe any revolute or prismatic joint as follows:

i. Link length (ai) - distance measured along xi axis from the point of intersection of xi axis with zi1 axis to the origin of frame $\{i\}$. 
ii. Link twist ( $\alpha \mathrm{i})$ - angle between zi-1 and zi axes measured about xi-axis in the right hand sense.

iii. Joint distance (di) - distance measured along zi-1 axis from the origin of frame $\{\mathrm{i}-1\}$ to intersection of xi axis with zi-1 axis.

iv. Joint angle $(\theta \mathrm{i})$ - angle between xi-1 and xi axes measured about the zi-1 axis in the right hand sense.

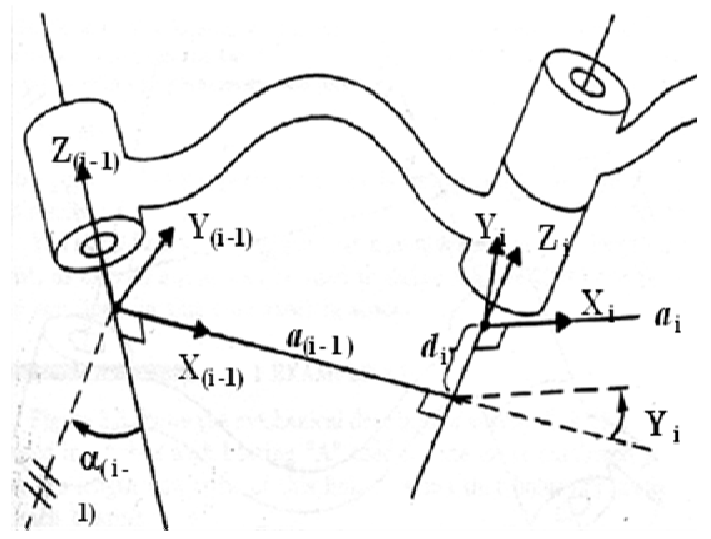

Figure 2. DH Conventions for frame assigning



Figure 3. D-H representation of Pravak Robot Arm

The kinematic model is shown in Figure 3 with frame assignments according to the Denavit \& Hartenberg (D-H) notations. The kinematic parameters according to this model are given in Table 2.

Table 2. D-H Parameter for Pravak Robot Arm

\begin{tabular}{ccccc}
\hline Joint & $\Theta_{\mathrm{i}}\left({ }^{0}\right)$ & $\alpha_{\mathrm{i}}\left({ }^{0}\right)$ & $\mathrm{a}_{\mathrm{i}}(\mathrm{mm})$ & $\mathrm{d}_{\mathrm{i}}(\mathrm{mm})$ \\
\hline 1 & $\Theta_{1}$ & -90 & 0 & 226 \\
2 & $\Theta_{2}$ & 0 & 179 & 0 \\
3 & $\Theta_{3}$ & 0 & 177 & 0 \\
4 & $\Theta_{4}$ & -90 & 0 & 0 \\
5 & $\Theta_{5}$ & 0 & 0 & 80 \\
\hline
\end{tabular}

\subsection{Kinematic relationship between adjacent links}

Once the DH coordinate system has been established for each link, a homogeneous transformation matrix can easily be developed considering frame $\{i-1\}$ and frame $\{i\}$. This transformation consists of four basic transformations as shown in Figure 4 and the joint link parameter as given in Table 1.

i. A rotation about zi- 1 axis by an angle $\theta \mathrm{i}$

ii. Translation along zi-1 axis by distance di

iii. Translation by distance ai along xi axis and

iv. Rotation by angle $\alpha$ i about xi axis $\mathrm{i}-1$

$\mathrm{Ti}=\mathrm{Tz}(\theta \mathrm{i}) \mathrm{Tz}(\mathrm{di}) \mathrm{Tx}(\mathrm{ai}) \mathrm{Tx}(\alpha \mathrm{i})$

$$
\begin{aligned}
{ }^{i-1} T_{i} & =\left[\begin{array}{cccc}
C \theta i & -S \theta i & 0 & 0 \\
S \theta i & C \theta i & 0 & 0 \\
0 & 0 & 1 & 0 \\
0 & 0 & 0 & 1
\end{array}\right]\left[\begin{array}{cccc}
1 & 0 & 0 & 0 \\
0 & 1 & 0 & 0 \\
0 & 0 & 1 & d i \\
0 & 0 & 0 & 1
\end{array}\right]\left[\begin{array}{cccc}
1 & 0 & 0 & a i \\
0 & 1 & 0 & 0 \\
0 & 0 & 1 & 0 \\
0 & 0 & 0 & 1
\end{array}\right]\left[\begin{array}{cccc}
1 & 0 & 0 & 0 \\
0 & C \alpha i & -S \alpha i & 0 \\
0 & S \alpha i & C \alpha i & 0 \\
0 & 0 & 0 & 1
\end{array}\right] \\
{ }^{i-1} T_{i} & =\left[\begin{array}{cccc}
C \theta i & -S \theta i C \alpha i & S \theta i S \alpha i & a i C \theta i \\
S \theta i & C \theta i C \alpha i & -C \theta i S \alpha i & a i S \theta i \\
0 & S \alpha i & C \alpha i & d i \\
0 & 0 & 0 & 1
\end{array}\right]
\end{aligned}
$$


where $\mathrm{S} \theta_{\mathrm{i}}=\sin \theta_{\mathrm{i}}, \mathrm{C} \theta_{\mathrm{i}}=\cos \theta_{\mathrm{i}}, \mathrm{S} \alpha_{\mathrm{i}}=\sin \alpha_{\mathrm{i}}, \mathrm{C} \alpha_{\mathrm{i}}=\cos \alpha_{\mathrm{i}}, \mathrm{S}_{\mathrm{ijk}}=\sin \left(\theta_{\mathrm{i}}+\theta_{\mathrm{j}}+\theta_{\mathrm{k}}\right), \mathrm{C}_{\mathrm{ijk}}=\sin \left(\theta_{\mathrm{i}}+\theta_{\mathrm{j}}+\theta_{\mathrm{k}}\right)$

The overall transformation matrix, ${ }^{0} T_{5}={ }^{0} T_{1}{ }^{* 1} T_{2}{ }^{* 2} T_{3}{ }^{*} T_{4}{ }^{*}{ }^{4} T_{5}$

$$
\begin{aligned}
& { }^{0} T_{1}=\left[\begin{array}{cccc}
c_{1} & 0 & s_{1} & a_{1} c_{1} \\
s_{1} & 0 & -c_{1} & a_{1} s_{1} \\
0 & 1 & 0 & d_{1} \\
0 & 0 & 0 & 1
\end{array}\right] \quad{ }^{1} T_{2}=\left[\begin{array}{cccc}
c_{2} & -s_{2} & 0 & a_{2} c_{2} \\
s_{2} & c_{2} & 0 & a_{2} s_{2} \\
0 & 0 & 1 & 0 \\
0 & 0 & 0 & 1
\end{array}\right] \quad{ }^{2} T_{3}=\left[\begin{array}{cccc}
c_{3} & -s_{3} & 0 & a_{3} c_{3} \\
s_{3} & c_{3} & 0 & a_{3} s_{3} \\
0 & 0 & 1 & 0 \\
0 & 0 & 0 & 1
\end{array}\right] \\
& { }^{3} T_{4}=\left[\begin{array}{cccc}
c_{4} & 0 & s_{4} & 0 \\
s_{4} & 0 & -c_{4} & 0 \\
0 & 1 & 0 & 0 \\
0 & 0 & 0 & 1
\end{array}\right] \quad{ }^{4} T_{5}=\left[\begin{array}{cccc}
c_{5} & -s_{5} & 0 & 0 \\
s_{5} & c_{5} & 0 & 0 \\
0 & 0 & 1 & d_{5} \\
0 & 0 & 0 & 1
\end{array}\right] \\
& { }^{o} T_{5}=\left[\begin{array}{cccc}
c_{1} c_{234} c_{5}+s_{1} s_{5} & c_{1} c_{234} s_{5}+s_{1} c_{5} & c_{1} s_{234} & c_{1}\left(d_{5} s_{234}+a_{3} c_{23}+a_{2} c_{2}+a_{1}\right) \\
s_{1} c_{234} c_{5}-c_{1} s_{5} & -s_{1} c_{234} s_{5}-c_{1} c_{5} & s_{1} s_{234} & s_{1}\left(d_{5} c_{234}+a_{3} s_{23}+a_{2} c_{2}+a_{1}\right) \\
s_{234} c_{5} & -s_{234} s_{5} & -c_{234} & \left(-d_{5} c_{234}+a_{3} s_{23}+a_{2} s_{2}+d_{1}\right) \\
0 & 0 & 0 & 1
\end{array}\right] \\
& { }^{0} T_{5}=T_{e}=\left[\begin{array}{cccc}
n_{x} & o_{x} & a_{x} & p_{x} \\
n_{y} & o_{y} & a_{y} & p_{y} \\
n_{z} & o_{z} & a_{z} & p_{z} \\
0 & 0 & 0 & 1
\end{array}\right]
\end{aligned}
$$

Where, Te is end-effector transformation matrix.

This kinematic model can also be expressed by 12 equations as:

$$
\begin{aligned}
& \mathrm{nx}=\mathrm{c} 1 * \mathrm{c} 234 * \mathrm{c} 5+\mathrm{s} 1 * \mathrm{~s} 5 \\
& \mathrm{ny}=\mathrm{s} 1 * \mathrm{c} 234 * \mathrm{c} 5-\mathrm{c} 1 * \mathrm{~s} 5 \\
& \mathrm{nz}=\mathrm{s} 234 * \mathrm{c} 5 \\
& \mathrm{ox}=\mathrm{c} 1 * \mathrm{c} 234 * \mathrm{~s} 5+\mathrm{s} 1 * \mathrm{c} 5 \\
& \mathrm{oy}=-\mathrm{s} 1 * \mathrm{c} 234 * \mathrm{~s} 5-\mathrm{c} 1 * \mathrm{c} 5 \\
& \mathrm{oz}=-\mathrm{s} 234 * \mathrm{~s} 5 \\
& \mathrm{ax}=\mathrm{c} 1 * \mathrm{~s} 234 \\
& \text { ay }=\mathrm{s} 1 * \mathrm{~s} 234 \\
& \mathrm{az}=-\mathrm{c} 234 \\
& \mathrm{px}=\mathrm{c} 1 *(\mathrm{~s} 234 * \mathrm{~d} 5+\mathrm{a} 3 * \mathrm{c} 23+\mathrm{a} 2 * \mathrm{c} 2+\mathrm{a} 1) \\
& \mathrm{py}=\mathrm{s} 1 *(\mathrm{~s} 234 * \mathrm{~d} 5+\mathrm{a} 3 * \mathrm{c} 23+\mathrm{a} 2 * \mathrm{c} 2+\mathrm{a} 1) \\
& \mathrm{pz}=-\mathrm{c} 234 * \mathrm{~d} 5+\mathrm{a} 3 * \mathrm{~s} 23+\mathrm{a} 2 * \mathrm{~s} 2+\mathrm{d} 1
\end{aligned}
$$

\section{RESULT}

For the homeposition, a program in MATLAB 2007 is made and its output is compared with the experimental result as follows.

Experimental Result:

$$
[0
$$

177

325

1]

Matlab Program Output:

$[0$ 


\section{CONCLUSION}

The forward kinematic analysis of 5-dof Pravak Robot is investigated. The mathematical model is prepared and solved for positioning and orienting the end effector by preparing a programme in MATLAB 2007. The experimental and theoretical results are approximately same. Hence this proves the utility of the Pravak robot arm as an educational tool for undergraduate robotics courses.

\section{REFERENCES}

[1] Pravak Robot User's Manual

[2] Paul RP. Robot Manipulators: Mathematics, Programming and Control. Cambridge, MIT Press. 1981.

[3] Paul RP, Shimano B, Mayer GE. Kinematic control equation for simple manipulators. IEEE Trans. Syst. Man. Cybern. SMC-11. 198; 449-455.

[4] Featherstone R. Position and velocity transformations between robot end-effector cordinates and joint angles. Int. J. Robotics Res. 1983; 2: 35-45.

[5] Lee CSG. Robot arm kinematics, dynamics, and control. Computer. 1982; 62-80.

[6] RK Mittal, J Nagrath. Robotics and Control. Tata McGraw-Hill. 2005; 76-81.

[7] Saeed B Niku. Introduction to Robotics. Pearson Prentice Hall. 2008; 81-85.

\section{BIOGRAPHIES OF AUTHORS}

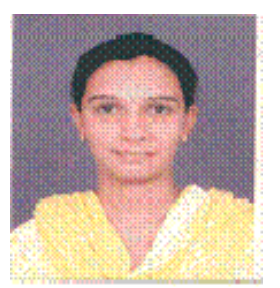

Ms. Jolly Shah is a Research Scholar at National Institute of Technology, Kurukshetra, India. She is Mechanical Engineering graduate from Birla Vishwakarma Mahavidyalaya, Vallabhvidyanagar, Gujarat, one of the premier institutes in the western India. She has obtained his M.E. degree in the field of Machine Design with Gold Medal and topper in university from Birla Vishwakarma Mahavidyalaya, Vallabhvidya nagar, Gujarat in the year 2005. Her area of research is Robotics. She has published 9 papers in conferences. She is associated with the Institution of Engineers (India) and Indian Society of Technical Education (ISTE).



Dr. S.S.Rattan is a professor and former head of the Mechanical department at National Institute of Technology, Kurukshetra, India. His area of interest are Tribology, Fluid Engineering \& Machine Design. He received "The Sir Rajendra Nath MookerjeeMemorial Prize" for the best paper in the Journal Of Institute Of Engineers (Mech.Division) for year 2002. He is also Author of books of Theory of Machines (TMH) \& Fluid Mechanics and Machinery (Khanna Publishers). Total no. of Research Publications: 29

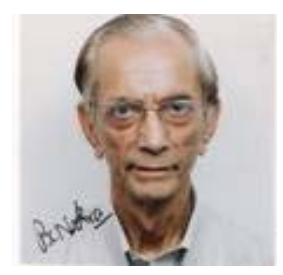

Professor B.C.Nakra worked as a Professor in Mechanical Engineering at Indian Institute of Technology, Delhi for several years before joining ITM Gurgaon in 2006. At IIT Delhi, he held B.H.E.L. Chair and Ram Rajindra Malhotra Emeritus Chair, in addition to having served as Head of Mechanical Engineering Dept., Head and joint Professor, Industrial Tribology, Machine Dynamics and Maintenance Engineering Centre and Head Instrument Design \& Development Centre. He was Dean, U.G. studies, Deputy Director (Faculty) and Offg. Director at IIT Delhi. During 2001-2003, he also worked as Professor of Eminence at Netaji Subhash Institute of Technology, Delhi University. Presently, he is Professor of Eminence at ITM University, Gurgaon. He has been involved in teaching and research in the areas of Vibration control and analysis of machines and structures, Condition monitoring and diagnostic maintenance, Dynamic design, Mechatronics, Instrumentation and Automatic Controls. He has guided $21 \mathrm{Ph}$. Ds, 67 M.Tech thesis and has published 3 books, 174 research papers in reputed journals and Conference Proceedings, in addition to 68 technical reports. Based on his research contributions, he has been elected Fellow of Indian National Science Academy (FNA), Indian National Academy of Engineering (FNAE), Academy of Sciences (F.A.Sc) National Academy of Sciences (FNASc.) and of World Innovation Foundation (FWIF). He has received awards like C.V.Raman award of Acoustical Society of India, Eminent Engineer award of Institution of Engineers India, G.P. Chatterjee Memorial award from INSA, Distinguished Mechanical Engineering Educator from ISME etc. He has served on the editorial board of Sadhana, Journal, PINSA, Journal, Tribology International, Maintenance Journal, International Journal of Applied Maths and Mechanics.He has been consultant to leading industries and organizations including B.H.E.L, Indian Railways, ONGC, SAIL, EIL, BEL, Eicher, Escorts, Mahindra \& Mahindra, Hero Motors, NSTL etc. in addition to lecturing in continuing education programmes and has been involved in several sponsored research projects of DST, ARDB, CSIR and others. 